TP Periodica Polytechnica Chemical Engineering

62(1), pp. 21-31, 2018

https://doi.org/10.3311/PPch.10550

Creative Commons Attribution (i)

RESEARCH ARTICLE

\section{Silica Sol-gel Coatings with Improved Light Transmittance and Stability}

\author{
Lenke Kócs ${ }^{1}$, Emőke Albert ${ }^{1}$, Borbála Tegze ${ }^{1}$, Márta Kabai-Faix ${ }^{1}$, \\ Csaba Major ${ }^{2}$, András Szalai ${ }^{2}$, Péter Basa ${ }^{3}$, Zoltán Hórvölgyi ${ }^{*}$
}

Received 27 January 2017; accepted after revision 15 February 2017

\begin{abstract}
Silica sol-gel coatings on different substrate materials and their use as model systems have been studied. Mesoporous silica coatings with thicknesses of $85-135 \mathrm{~nm}$ and porosity of $18-37 \%$ were prepared by dip-coating on polycarbonate and glass substrates. In order to eliminate the shrinkage of the porous structure acid or base vapour treatment was applied. Thickness and refractive index of the coatings were determined by analysing the transmittance spectra of the samples. Ellipsometric porosimetry measurements were carried out to determine the porosity, pore radius distribution, thickness and refractive index. The thickness of the samples was further confirmed by scanning electron microscopy. The adsorption capacity of the porous coatings was also studied by dye impregnation tests. The temporal stability of the samples was investigated by $U V$-Vis spectrometry and it was found that the advantageous optical properties $\left(T_{\max }=98-99 \%\right)$ of the samples remained constant even after a 1 year storage period.
\end{abstract}

\section{Keywords}

sol-gel coating, improved light transmittance, long-term optical stability, network strengthening, porosity

\footnotetext{
${ }^{1}$ Department of Physical Chemistry and Materials Science, Centre for Colloid Chemistry,

Faculty of Chemical Technology and Biotechnology, Budapest University of Technology and Economics, H-1521 Budapest, Budafoki út 6-8, Hungary

${ }^{2}$ Hungaro Lux Light Ltd.,

H-1171 Budapest, Strázsahegyi dủlő 7, Hungary

${ }^{3}$ Semilab Semiconductor Physics Laboratory Co. Ltd.,

H-1117 Budapest, Prielle Kornélia utca 2, Hungary

*Corresponding author, e-mail: zhorvolgyi@mail.bme.hu
}

\section{Introduction}

Porous silica sol-gel coatings with improved light transmittance (anti-reflective coating $=\mathrm{AR}$ ) are increasingly popular in various research areas and applications. One of their most important basic requirements is that they maintain their optical stability during the time of their utilization. Attaining sustainable long-lasting porous thin films with AR properties is a major challenge, especially in lighting technologies and in related applications, for instance solar energy conversion [1]. There are numerous requirements in order to avoid the porous system filling with pollutants from the air or the aging process during the utilization (collapsing of structure).

Anti-reflective coatings can be single-, double-, or multilayers with gradient or constant refractive index, using coatings or surface texturing on the target surface. Antireflective coatings can be fabricated with nanophysical (chemical vapour deposition, etching, lithography) or with nanochemical methods (solgel process, etc.) [2-4].

Sol-gel process is one of the commonly used wet chemical methods to prepare AR coatings. The sol-gel techniques are relatively low-cost, the reaction conditions are mild, and moreover the composition and structure of coatings can be easily controlled. The first step of the preparation is the hydrolysis of a suitable silane compound (e.g. alkoxy-silane) in aqueous or alcoholic medium which can be initiated by base or acid catalysis. The hydrolysis followed by polycondensation reaction results in the formation of colloidal particles, macromolecules and/or microphases (precursor sol or solution). Mainly microphases form in case of base catalysis (referred as base catalyzed silica-particles or layers) while using acid catalysis (referred to as acid catalyzed system) polysiloxane chains form in the precursor solution. Deposition of precursor sol onto a solid substrate by dip-coating (or spin-coating process, etc.) results in a thin liquid film on the solid surface, and after drying and/or suitable heat treatment the sol-gel coating is acquired. Mesoporous coatings can be prepared by depositing microphases (the deposited layer will contain several voids among the particles) or by using micellar templates to form the pore system. Strengthening the sol-gel network by post-treatments 
is required in long-term applications. One of the most commonly used treatment methods is placing the samples under an ammonia vapour atmosphere [5-8]. A number of studies have focused specifically on the hardening effect of the ammonia treatment. Therefore, the most actively investigated area in this topic deals with sol-gel porous coatings used in high-power laser applications. The hardening effect was first reported by Floch and Belleville [9]. They have found that porous silica sol-gel coatings synthesized with base-catalyzed reactions exposed to ammonia vapours or immersed into alkaline ethanolic solutions gained adhesive- and abrasion resistance on glass substrates. As a consequence of the ammonia treatment the thickness diminished and the transmittance spectra showed a blue shift. They explained these findings with an increased amount of siloxane bridges and H-bonds between the adjacent silica particles creating a strengthened (and shrunk) crosslinked network.

Using ammonia treatment Shen and $\mathrm{Li}$ [7] were able to increase the hardness of silica sol-gel particles against the laser-induced damage threshold. They observed enlarged pore size and decreased thickness as consequences of the treatment. All of the above-mentioned researches focused on increasing the physical stability of base catalysed silica particle coatings. Grosso et al. have found that ammonia treatment can replace the thermal curing of acid catalysed mesoporous silica thin films prepared by micellar templates [11]. They concluded that the $\mathrm{OH}^{-}$takes part in catalytic reactions at the micelle/silica interfaces and improves the stability of the pore system, but the temporal behaviour of the system was not investigated.

The stability of the optical properties during storage has not been fully explored.

The purpose of this work was to prepare mesoporous silica coatings by single layer deposition to improve the long-term stability of the light transmittance. The samples were strengthened in ammonia or in hydrochloric acid vapour atmosphere. Two optically transparent substrates (polycarbonate and microscope glass slides) were coated by sol-gel method. The effect of the acid or base treatment and the treatment time on the pore system was investigated. In certain cases, the open porosity and structure of the coatings were characterized using ellipsometric porosimetry and dye impregnation tests. The adsorption of a cationic dye in the pore system was characterized by UV-Vis spectrophotometry method. The optical stability behaviour of the silica coatings was monitored by measuring the transmittance spectra for one year.

\section{Experimental section}

\subsection{Materials}

Tetraethyl orthosilicate (TEOS, Acros Organics reagent, grade 98\%), 2-propanol (Reanal reagent, grade $>99.7 \%$ ), Pluronic PE10500 (BASF reagent), Pluronic P123 (BASF reagent), hydrochloric acid ( $\mathrm{HCl}$, Fluka reagent, purum, 37\%) and deionized water $(18.2 \mathrm{M} \Omega \cdot \mathrm{cm}$, purified with a Millipore Simplicity 185 Filtration System) were used to synthesize silicon-dioxide precursor sol.

Microscope glass slides $(76 \times 26 \times 1 \mathrm{~mm}$, Thermo Scientific, Menzel-Gläser), and polycarbonate sheets $(\mathrm{PC}, 50 \times 25 \times 2.1 \mathrm{~mm}$ LEXAN $^{\mathrm{TM}}$, XL102UV Sheet) were used as substrate materials.

(3-aminopropyl)triethoxysilane (APS, Reanal reagent, grade 97\%) was used for the pre-treatment of PC sheets for better adhesion. Ammonia solution (Reanal reagent, a.r., grade $25 \%$ ), and hydrochloric acid solution were used for the network strengthening. Rhodamine 6G dye (R6G, Sigma-Aldrich, grade $95 \%$ ) was used for dye impregnation tests. All chemicals were used without further purification.

\subsection{Silica sol preparation}

The silica sol was prepared by the acid catalyzed controlled hydrolysis of TEOS. The molar ratio of the components was TEOS: 2-PrOH: $\mathrm{HCl}: \mathrm{H}_{2} \mathrm{O}: \mathrm{PE} 10500=1:$ 14.49: 0.01: 5.61: 0.01, and TEOS: 2-PrOH: $\mathrm{HCl}: \mathrm{H}_{2} \mathrm{O}: \mathrm{P} 123=1:$ 14.49: 0.01: 5.61: 0.01 .

In the first step, Pluronic PE10500 or Pluronic P123 surfactant and hydrochloric acid were added to the alcoholic medium, and it was stirred until the complete dissolution of the surfactant at room temperature. To this TEOS was added and the mixture was stirred for further 30 minutes.

The as-prepared silica sol was stored at room temperature before being used. In all cases, only one day aged sols were applied for coatings preparation.

\subsection{Sample preparation}

Glass slides were soaked in $1 \%$ detergent solution, rinsed with distilled water, neutralized with dilute sulphuric acid, rubbed with 2-propanol moistened cellulose wadding, then rinsed with 2-propanol, and washed with deionized water. PC substrates were ultrasonicated in $1 \%$ detergent solution for 5 minutes, and then rinsed with distilled water. All substrates were dried at $70{ }^{\circ} \mathrm{C}$ in a drying oven (Memmert UNB 400).

\section{Pre-treatment of substrates}

PC substrates were immersed into a $1 \%$ solution of the coupling agent (3-aminopropyl)triethoxysilane in 2-propanol for $1 \mathrm{~h}$.

\section{Layer deposition}

Sol-gel films were prepared from the precursor sol by dip-coating method (dip coater, Plósz Engineering Ltd., Hungary) on PC and glass substrates. Cleaned and dried substrates were immersed into the precursor sol and then withdrawn at a constant speed. Withdrawal speeds were $15 \mathrm{~mm} / \mathrm{min}$ for glass, and $10 \mathrm{~mm} / \mathrm{min}$ for PC substrates. For ellipsometric porosimetry measurements withdrawal speeds of $10 \mathrm{~mm} / \mathrm{min}$ and $30 \mathrm{~mm} / \mathrm{min}$ were used for samples on glass substrates.

\section{Network strengthening treatments}

The as-deposited coatings were kept in a desiccator that was filled with ammonia or hydrochloric acid vapours (in 
equilibrium with $2 \mathrm{M}$ ammonia solution or with $6 \mathrm{M}$ hydrochloric acid solution) for $1 \mathrm{~h}$ (in case of hydrochloric acid, and for samples on glass substrates in ammonia) and $3 \mathrm{~h}$ (in case of samples on PC substrates in ammonia). The vapour treatments were performed at room temperature. The acid or base vapourtreated lyogels were heat treated with the following method. A number of coatings on glass substrates were calcined in a muffle furnace (Nabertherm B170) at $480{ }^{\circ} \mathrm{C}$ for $1 \mathrm{~h}$ with a heating rate of $15{ }^{\circ} \mathrm{C} / \mathrm{min}$. The coatings on PC substrates and the remaining samples on glass substrates were dried in a drying oven at $120^{\circ} \mathrm{C}$ for $13 \mathrm{~h}$. Fig. 1 summarizes the steps of the sample preparation.

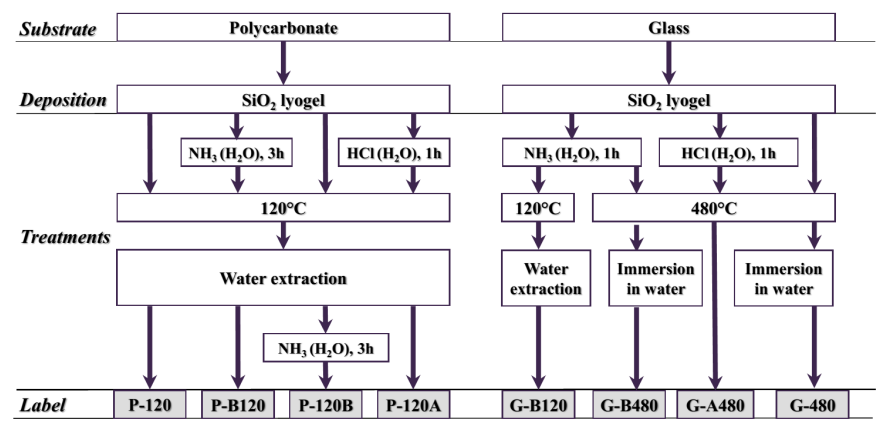

Fig. 1 Block diagram of the preparation steps of the porous silica coatings on PC (P) and glass (G) substrates, heat treated at $120^{\circ} \mathrm{C}(120)$ and $480{ }^{\circ} \mathrm{C}$ (480); B refers to the base, and similarly A refers to the acid vapour treatment.

The acid and base vapour treatments take place at room temperature.

\subsection{Characterization of the samples}

The presence of coupling agents

Coupling agent ((3-aminopropyl)triethoxysilane) was applied to eliminate the disadvantageous effects caused by the significant difference between the thermal expansion of the polymer substrate and the inorganic-oxide materials [12]. The presence of the coupling agent in the samples was confirmed by $\mathrm{X}$-ray photoelectron spectroscopy measurements. The results show $0.6 \% \mathrm{Si}$ and $0.7 \% \mathrm{~N}$ on the surface. This analysis indicates the presence of the APS molecules on the PC surface.

Studying light transmittance of coated samples by UV-Vis spectrophotometry method

Transmittance spectra of the prepared coatings on glass and on PC substrates were recorded using a UV-Vis spectrophotometer (Analytic Jena, Specord ${ }^{\circledR} 200$ ). The transmittance spectra were measured in the wavelength range of $400-1100 \mathrm{~nm}$, with slit $1 \mathrm{~nm}$, integration time $0.20 \mathrm{~s}$, scanning speed $5 \mathrm{~nm} / \mathrm{s}$, and resolution $1 \mathrm{~nm}$.

\section{Analysis of light transmittance spectra}

From the analysis of transmittance spectra, the average transmittance increment (ATI), the maximum light transmittance $\left(\mathrm{T}_{\max }\right)$, layer thickness $(\mathrm{d})$, and refractive index $(\mathrm{n})$ were determined. The ATI is calculated from the difference of the integrated area of the transmittance spectra measured on the coated sample $\left(\mathrm{A}_{\text {sample }}\right)$ and the substrate $\left(\mathrm{A}_{\text {substrate }}\right)$ in the investigated wavelength range $(410-780 \mathrm{~nm})$ as described by Eq. (1)

$$
A T I=\frac{A_{\text {sample }}-A_{\text {substrate }}}{780 \mathrm{~nm}-410 \mathrm{~nm}}
$$

The maximum light transmittance value was selected in the range between $410 \mathrm{~nm}$ and $780 \mathrm{~nm}$. Despite the fact that the transmittance spectra were recorded between $400 \mathrm{~nm}$ and $1100 \mathrm{~nm}$, the aforementioned region is particularly relevant in practical applications (e.g. LED street lighting).

The refractive index was determined as 1.585 for PC and 1.517 for glass substrates at $632.8 \mathrm{~nm}$. The effective refractive index of the coating on the transparent substrate was determined at $632.8 \mathrm{~nm}$ by using an optical fitting model. The fitting procedure was described in detail in our previous papers [13-15]. The fitting procedure uses the Levenberg - Marquardt algorithm [16]. The porosity of coatings was estimated using the Lorentz-Lorenz formula [18-20].

Studying the pore structure of coatings by ellipsometric porosimetry (EP)

In certain cases, ellipsometric porosimetry measurements were carried out to determine the (open) porosity, pore size distribution, porosity, thickness and refractive index of the coatings. G-120 and G-480 type samples on glass substrates were characterized by a Semilab's PS-2000 apparatus. The measurements were carried out in the wavelength range of $275-800 \mathrm{~nm}$. The thickness and the effective refractive index of the coatings were determined by applying the Cauchy model. The adsorption isotherms were taken at $300 \mathrm{~K}$, and 2-propanol was used as the adsorptive material.

Studying the pore structure of coatings by cationic dye impregnation tests

The main objective of cationic dye impregnation tests was to get indirect information about the effect of ammonia treatment on the pore structure by studying the adsorption capacity of the coatings. Coated glass substrates (G-120, G-B120, G-480, and G-B480) were immersed into $10^{-4} \mathrm{M}$ aqueous impregnating solution of R6G. After 1 minute of impregnation dye molecules in the pores reached the equilibrium accumulation. The samples were rinsed with distilled water to remove the excess dye from the surface. The absorbance of impregnated samples was measured with UV-Vis spectrophotometer between $420 \mathrm{~nm}$ and $600 \mathrm{~nm}$.

Characterization of coatings by scanning electron microscopy (SEM)

In certain cases, the thickness of the porous silica coatings was further confirmed by scanning electron microscopy. Cross sectional images of samples on glass substrates (G-B480) were taken using a LEO 1540 XB type scanning electron microscope with $3 \mathrm{kV}$ acceleration voltage. Before the measurements samples were coated with gold using a sputter coater. 

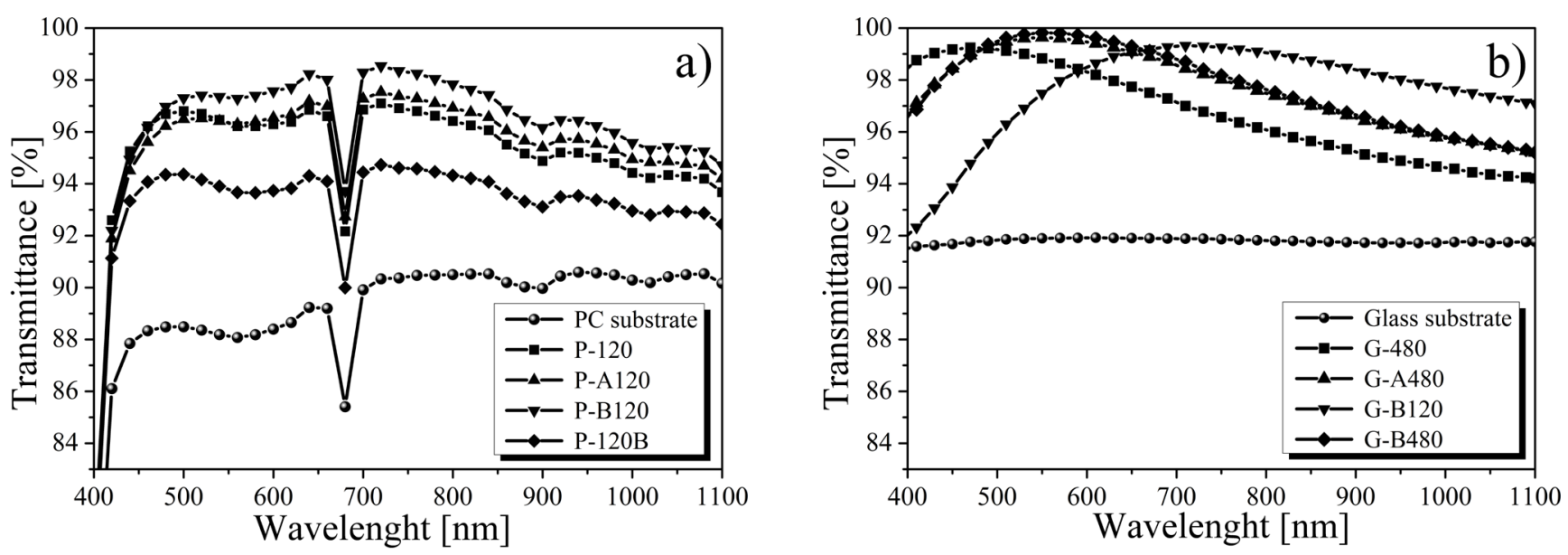

Fig. 2 Transmittance spectra of the bare $\mathrm{PC}$ substrate and the untreated $\mathrm{SiO}_{2}$ sample $(\mathrm{P}-120)$, hydrochloric acid treated $\mathrm{SiO}{ }_{2}$ sample $(\mathrm{P}-\mathrm{A} 120)$, ammonia treated $\mathrm{SiO}_{2}$ sample (P-B120), and ammonia treated $\mathrm{SiO}_{2}$ sample (P-120B) on PC substrate (a), and the transmittance spectra of the bare glass substrate and the untreated $\mathrm{SiO}_{2}$ sample (G-480), hydrochloric acid treated $\mathrm{SiO}_{2}$ sample (G-A480), ammonia treated $\mathrm{SiO}_{2}$ sample at $120{ }^{\circ} \mathrm{C}(\mathrm{G}-\mathrm{B} 120)$, and ammonia treated $\mathrm{SiO}{ }_{2}$ sample at $480^{\circ} \mathrm{C}$ (G-B480) on glass substrate (b). All ammonia treatments were carried out on the lyogel forms with the exception of samples P-120B. In the latter case samples were treated in ammonia vapours after extraction. The absorption peak appearing at $682 \mathrm{~nm}$ in (a) is attributed to the absorption of a UV protection additive found in the PC substrate.

\section{Results and discussion}

The results are reported in the following sections. In the first part the results are interpreted and discussed for the as-prepared samples. Investigations for the long-term optical behaviour are shown in the second part.

\subsection{Properties of as-prepared samples}

Without pre-treatment with a coupling agent, porous silica coatings on polycarbonate substrates show micro-cracking after the heat treatment. In comparison, crack free, homogeneous surfaces formed when the samples were pre-treated with the coupling agent.

This advantageous effect of the pre-treatment is due to the presence of APS molecules which ensures better adhesion between the coating and the PC surface. During pre-treatment the silica coating is covalently bonded to the substrate due to the aminolysis mechanism between the APS and PC [12]. The effect of the ammonia and hydrochloric acid vapour treatment on the transmittance of samples was studied. In the case of ammonia treatments, two different methods were carried out. In the first method, the base vapour treatment was applied after the heat treatment and extraction of the coatings. In the second method, the base vapour treatment was carried out on the lyogel form still containing the templates, before the heat treatment.

\section{UV-Vis transmittance spectra of the prepared systems}

Fig. 2 demonstrates the differences in the light transmittance caused by the different treatments. These spectra were measured after the final preparation step. It can be observed that the post-extraction ammonia vapour treatment has an unfavourable effect (Fig. 2a, P-120B), as the light transmittance is the lowest in this case. In comparison, all other spectra show a remarkably high light transmittance. Therefore, in further experiments, only the different network strengthening treatments of the lyogels were investigated.

\section{Analysis of the UV-Vis transmittance spectra}

The ATI and $\mathrm{T}_{\text {max }}$ values, the fitted parameters ( $\mathrm{n}$ and $\mathrm{d}$ ), and the calculated porosity are presented in Table 1 . For every transmittance spectrum, a fitting procedure was performed in order to obtain the layer thickness and the effective refractive index. The accuracy of the optical fitting procedure is monitored by the reduced chi-squared values and the coefficient of determination $\left(\mathrm{R}^{2}\right)$ values. These values are in the range of $10^{-7}-10^{-8}$, and above 0.995 , respectively. Therefore, there is a good correspondence between measured and calculated spectra.

The effective refractive index of P-120B samples $(1.453 \pm 0.006)$ indicates no porosity after post extraction ammonia vapour treatment. This can be explained by the ammonia molecules inducing cross-links in the coating structure that results in its shrinkage, which leads to the deterioration of the light transmittance (Table 1). The light transmittance after the network strengthening of the lyogels with base vapours (P-B120 and G-B480) is higher than after the network strengthening with acid vapours (P-A120 and G-A480). Samples without any network strengthening (P-120 and G-480) show a lower light transmittance. On the basis of the high efficiency of this type of network strengthening treatment, hereinafter the vapour-treatments were applied on the lyogel form of the silica coatings.

Floch and Bellevile described that the alkaline treatment on silica particulate layer caused a decrease in the layer thickness [9]. This shrinking effect was not observed in our system. We obtained higher layer thicknesses when using acid treatment $(107 \pm 3 \mathrm{~nm}, \mathrm{P}-\mathrm{A} 120 ; 104 \pm 1 \mathrm{~nm}, \mathrm{G}-\mathrm{A} 480)$ or base treatment (112 $\pm 2 \mathrm{~nm}, \mathrm{P}-\mathrm{B} 120 ; 102 \pm 0 \mathrm{~nm}, \mathrm{G}-\mathrm{B} 480)$ compared to the 
Table 1 The average transmittance increment (ATI), maximum transmittance $\left(\mathrm{T}_{\max }\right)$, effective refractive index (n), layer thickness (d) and porosity values of different $\mathrm{SiO}_{2}$ coatings on PC and glass substrates obtained by UV-Vis spectrophotometry

\begin{tabular}{llllll}
\hline Samples & ATI [\%] & $\mathrm{T}_{\max }[\%]$ & $\mathrm{n}$ at $632.8 \mathrm{~nm}[-]$ & $\mathrm{d}[\mathrm{nm}]$ & Porosity [\%] \\
\hline G-480 & $6.34 \pm 0.07$ & $99.27 \pm 0.02$ & $1.300 \pm 0.004$ & $85 \pm 2$ & 28 \\
G-A480 & $7.07 \pm 0.03$ & $99.63 \pm 0.03$ & $1.283 \pm 0.003$ & $104 \pm 1$ & 36 \\
G-B120 & $6.01 \pm 0.24$ & $99.42 \pm 0.09$ & $1.302 \pm 0.003$ & $134 \pm 5$ & 32 \\
G-B480 & $7.22 \pm 0.04$ & $99.78 \pm 0.05$ & $1.267 \pm 0.004$ & $102 \pm 0$ & 37 \\
P-120 & $7.72 \pm 0.04$ & $97.17 \pm 0.11$ & $1.368 \pm 0.002$ & $101 \pm 1$ & 18 \\
P-A120 & $7.69 \pm 0.04$ & $97.57 \pm 0.16$ & $1.367 \pm 0.001$ & $107 \pm 3$ & 18 \\
P-B120 & $8.36 \pm 0.05$ & $98.57 \pm 0.03$ & $1.323 \pm 0.002$ & $112 \pm 2$ & 27 \\
P-120B & $5.36 \pm 0.08$ & $94.91 \pm 0.24$ & $1.453 \pm 0.006$ & $96 \pm 1$ & 0 \\
\hline
\end{tabular}

untreated coatings $(101 \pm 1 \mathrm{~nm}, \mathrm{P}-120 ; 85 \pm 2 \mathrm{~nm}, \mathrm{G}-480)$. Similarly the porosity values of network strengthened samples are equal or higher than those of the untreated samples (Table 1).

It is worth mentioning that in these cases the vapour treatment was applied directly on the lyogel forms of the samples. The lyogel forms still contain the micelles, which can withstand the constrictive impact of network strengthening vapour treatments and heat treatments. After examining the properties of the as-prepared samples the optimum systems (P-B120 and GB-480) were selected for long-term investigations, which ensure the highest ATI and the lowest $\mathrm{n}$ values. Additionally, the untreated coatings (P-120 and G-480) were selected as reference samples during further measurements. These selected samples were monitored by UV-Vis spectrophotometry for one year.

\section{Scanning electron microscopy studies}

Scanning electron microscopy was applied as an independent method to confirm the thickness of the silica coatings obtained from the optical fitting. Fig. 3 shows the cross-sectional view of the G-B480 single layer sample on glass substrate. The thickness defined with optical fitting for these type of samples $(102 \pm 0 \mathrm{~nm}$, Table 1) is in reasonable agreement with the one observed in the scanning electron image $(\sim 80 \mathrm{~nm})$.

\section{Ellipsometric porosimetry measurements}

The porous silica coatings on glass substrates that were not subjected to base or acid vapour network strengthening treatment have been investigated with ellipsometric porosimetry. Ellipsometric porosimetry is a coupled method in which the vapour adsorption can be studied step-by-step through spectroscopic ellipsometry. Furthermore, this technique provides the possibility of determining the optical properties of coatings as a spectroscopic ellipsometer at zero relative pressure of the adsorptive material.

The coatings were aged for one month before the measurement. The samples were measured under atmospheric conditions and in vacuum. The effective refractive index values obtained by spectroscopic ellipsometry in atmosphere (Table 2) are comparable with the optical fitted parameters (Table 1, G-480).

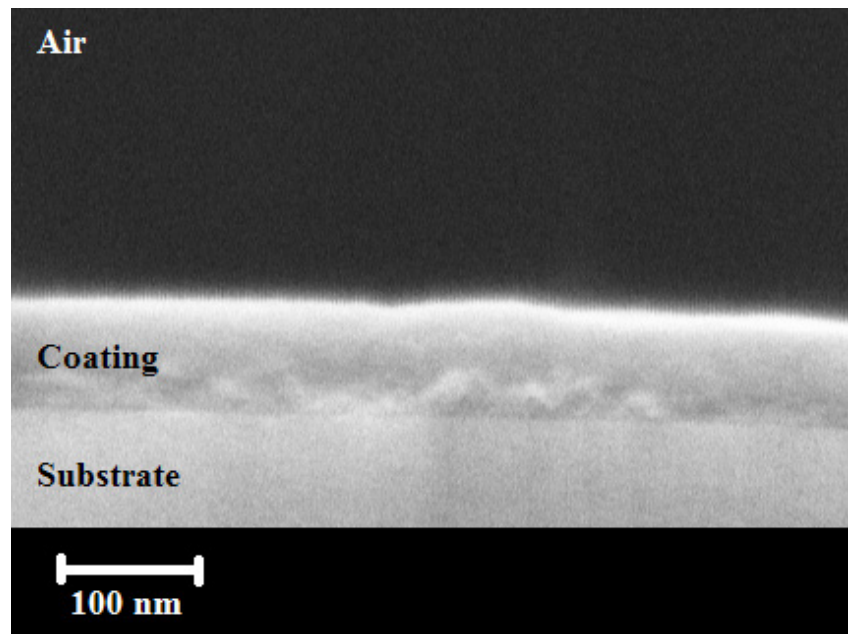

Fig. 3 Cross-sectional SEM image of ammonia-treated silica thin film (GB-480) on glass substrate.

Table 2 Layer thickness (d) and effective refractive index (n) values of untreated $\mathrm{SiO}_{2}$ coatings deposited on glass substrates (G-120 and G-480) obtained by spectroscopic ellipsometry measurements. The withdrawal speed was $10 \mathrm{~mm} / \mathrm{min}$ for G-120 samples and $30 \mathrm{~mm} / \mathrm{min}$ for G-480 samples.

\begin{tabular}{lllll}
\hline \multirow{2}{*}{ Samples } & \multicolumn{3}{c}{ Atmosphere } & \multicolumn{2}{c}{ Vacuum } \\
\cline { 2 - 5 } & $\mathrm{d}[\mathrm{nm}]$ & $\mathrm{n}$ at 632.8 nm [-] & $\mathrm{d}[\mathrm{nm}]$ & $\mathrm{n}$ at 632.8 nm [-] \\
\hline G-120 & $84.3 \pm 1.7$ & $1.3616 \pm 0.0032$ & $83.7 \pm 1.8$ & $1.3508 \pm 0.0019$ \\
G-480 & $110.6 \pm 8.3$ & $1.3267 \pm 0.0029$ & $110.2 \pm 9.1$ & $1.3122 \pm 0.0007$ \\
\hline
\end{tabular}



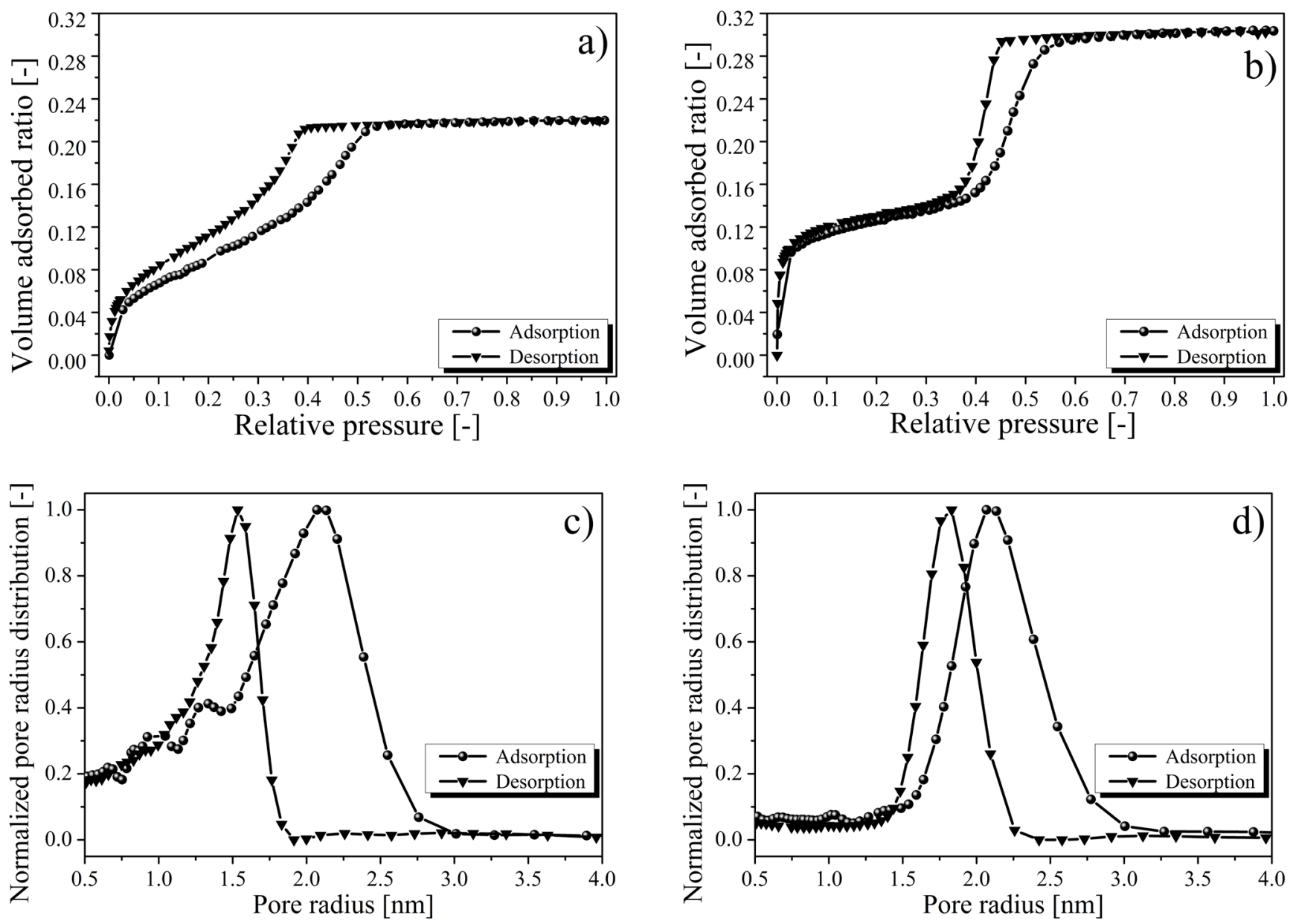

Fig. 4 2-propanol adsorption-desorption isotherms at $300 \mathrm{~K}$ measured by EP in the untreated G-120 (a) and G-480 (b) $\mathrm{SiO}_{2}$ coatings on glass substrates. Pore size distribution of untreated G-120 (c) and G-480 (d) $\mathrm{SiO}_{2}$ coatings on glass substrates determined by the modified Kelvin model applicable for mesopores.

The G-120 and G-480 samples have type IV adsorption isotherms according to the IUPAC classification, which is specific to mesoporous materials (Fig. 4a and 4b). The adsorption/ desorption hysteresis loops are type H1. The saturation appears at higher relative pressure for G-480 samples (Fig. $4 \mathrm{~b}, 0.45$ ) than for G-120 samples (Fig. 4a, 0.39) which is due to the presence of higher pore sizes in the G-480 samples.

The normalized pore radius distribution was determined from the volume adsorbed ratio by the modified Kelvin model and is presented in Fig. $4 \mathrm{c}$ and $4 \mathrm{~d}$. These results show that these thin films have narrow pore size distributions. The determined pore radius values obtained from the desorption isotherms are ca. $1.5 \mathrm{~nm}$ for G-120 and $1.8 \mathrm{~nm}$ for G-480 (Table 3).

The desorption isotherm shows that G-120 samples have a lower porosity $(22.20 \pm 0.44 \%)$, which may indicate that the pores are less interconnected within the film. Young-moduli of coatings were also determined. The Young modulus values of the coatings were calculated based on the film thickness isotherm measured during the ellipsometric porosimetry desorption cycle, using a combination of the Kelvin and the YoungLaplace equations, according to the method published in [20]. G-480 samples have a higher stiffness $(2.9 \pm 0.1 \mathrm{GPa})$ than

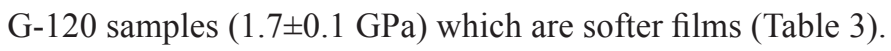

Table 3 Porosity and pore radius values determined by analysing the adsorption and desorption data, and Young's modulus values of untreated $\mathrm{SiO}_{2}$ coatings deposited on glass substrates (G-120 and G-480) obtained by ellipsometric porosimetry measurements. The pores were filled with 2-propanol.

\begin{tabular}{lllll}
\hline Samples & $\begin{array}{l}\text { Porosity } \\
{[\%]}\end{array}$ & $\begin{array}{l}\text { Pore radius } \\
\text { (ads.) }[\mathrm{nm}]\end{array}$ & $\begin{array}{l}\text { Pore radius } \\
\text { (des.) }[\mathrm{nm}]\end{array}$ & $\begin{array}{l}\text { Young's } \\
\text { modulus [GPa] }\end{array}$ \\
\hline $\mathbf{G - 1 2 0}$ & $22.20 \pm 0.44$ & $2.09 \pm 0.04$ & $1.53 \pm 0.00$ & $1.7 \pm 0.2$ \\
\hline G-480 & $30.15 \pm 0.35$ & $2.07 \pm 0.00$ & $1.80 \pm 0.05$ & $2.9 \pm 0.1$ \\
\hline
\end{tabular}

The porosity values of the G-480 samples determined with optical fitting $(28 \pm 0 \%)$ are in good agreement with those obtained from ellipsometric porosimetry adsorption data $(30.15 \pm 0.35 \%$, Table 3$)$. The porosity determined by the optical fitting gives the total porosity, while the ellipsometric porosimetry measurement provides the volume of the accessible pore system. Therefore, these results reveal that the entire pore system of the coatings is open and accessible to the small 2-propanol molecules.

\section{Dye impregnation}

Dye impregnation tests were carried out on silica coatings on glass substrates obtained from sols containing Pluronic P123 template (Section 2.2). Mesoporous coatings were impregnated with $10^{-4} \mathrm{M}$ solution containing R6G dye molecules (size of 

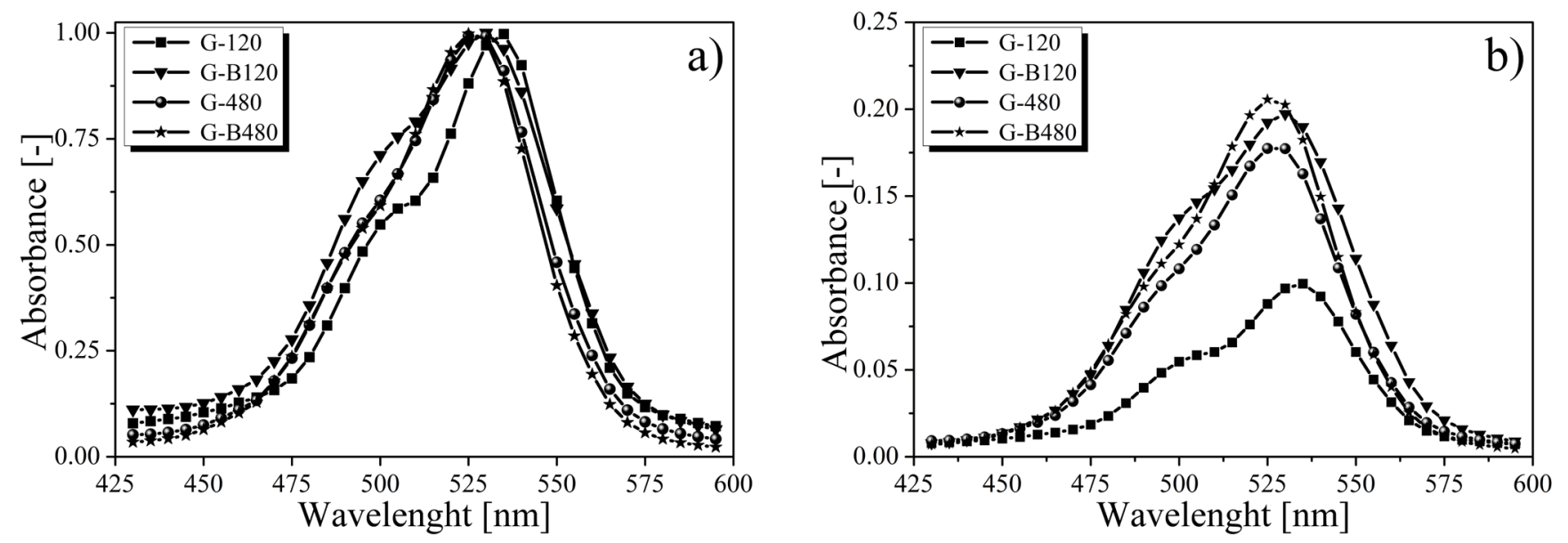

Fig. 5 Absorption spectra of the impregnated G-120, G-B120, G-480, and G-B480 samples normalized to 1 unit absorbance (a), and to 100 nm (b).

molecules $\sim 1 \times 1 \times 0.4 \mathrm{~nm}^{3}$ [21]) in order to define the accessibility of the pores [22]. The presented absorbance spectra (Fig. 5) were calculated as the difference of absorbance values of the sample measured after and before dye impregnation. The absorption spectra are normalized to absorbance unit of one (the scale adjusted so that for each curve maximal absorbance value equals one, Fig. 5a) and to $100 \mathrm{~nm}$ thickness of the coatings (absorbance values of samples with varying thicknesses adjusted to a theoretical coating of $100 \mathrm{~nm}$ thickness, Fig. 5b). The absorption peak detected at $530 \mathrm{~nm}$ is attributed to dye monomers, and the shoulder at $500 \mathrm{~nm}$ to the dye H-dimers [23]. The relative amount of monomer to dimer molecules of R6G in the pores can be analyzed as shown in Fig. 5a. G-B120 samples have the highest shoulder at $500 \mathrm{~nm}$, which indicates a higher amount of dimer molecules. Therefore, it appears that due to the ammonia treatment higher pore sizes are present in the coatings and dimerization can occur. Untreated samples prepared with a moderate temperature drying step (G-120) show the lowest amount of dye adsorbed as seen in Fig. 5b. The results show that both the ammonia and the higher temperature heat treatments result in the increase of the adsorbed amount of dye molecules. These findings are in good agreement with the calculated high porosity values (Table 3, G-480) obtained for ammonia treated systems and for those which were treated at higher temperature.

\subsection{Light transmittance of samples in a one-year time period}

Transmittance spectra of the silica coatings on transparent substrates were measured every month for one year. The representative transmittance spectra after 1,60,120,240, and 360 days of storage are presented in Fig. 6. This long-term monitoring of temporal changes in light transmittance is rarely found in other studies, even though investigating the temporal stability of AR coatings has great importance in practical applications. In Fig. 6, the first two charts show the transmittance spectra of the porous $\mathrm{SiO}_{2}$ coatings without any network strengthening (samples
P-120 and G-480; Fig. 6a and 6b). The other two charts show the transmittance spectra of the $\mathrm{SiO}_{2}$ coatings network strengthened in lyogel form with ammonia both on polycarbonate and on glass substrates (P-B120 and G-B480; Fig. 6c and 6d).

Throughout one year of storage a gradual decrease can be observed in the transmittance of the untreated P-120 and G-480 samples. This decrease in the transmittance values is more significant in the case of the samples on PC substrates than for the samples on glass substrates, presumably due to the lower temperature of the heat treatment. Light transmittance has reached its minimum value after 120 days, and further decline was not observed (Fig. 6a and 6b). When ammonia network strengthening is used, in the case of coatings on PC (P-B120) the transmittance of the samples shows no noticeable changes during the measured interval (Fig. 6c). In contrast, when glass was used as substrate, the light transmittance of G-B480 shows noticeable decrease after four months of storage (Fig. 6d).

Analysis of transmittance spectra: the maximum transmittance $\left(T_{\max }\right)$ and the average transmittance increment (ATI) values

The systematically measured maximum transmittance values clearly show that the ammonia-treated coating is stable for both P-B120 and G-B480 samples (Fig. 7), though a slight decrease can be observed for glass substrates after four months of storage.

Fig. $7 \mathrm{a}$ and $7 \mathrm{~b}$ show the calculated values of the average transmittance increment for samples on both polycarbonate and glass substrates. As the results show, the observed values are influenced by the type of substrate material. The ATI values of the same porous silica coatings are higher on PC $(8.36 \pm 0.05 \%$ for P-B120, and $7.65 \pm 0.12 \%$ for P-120) than on glass substrates $(7.22 \pm 0.04 \%$ for G-B 480 , and $6.34 \pm 0.07 \%$ for G- 480$)$. Moreover, the ammonia treatment has an advantageous effect on the optical properties of the porous silica coatings. The light transmittance remained constant in the whole measured wavelength range $(410-780 \mathrm{~nm})$ even after 1 year of storage when PC was used as substrate. The ATI values of G-B480 show continuous decrease (observable after four months of 

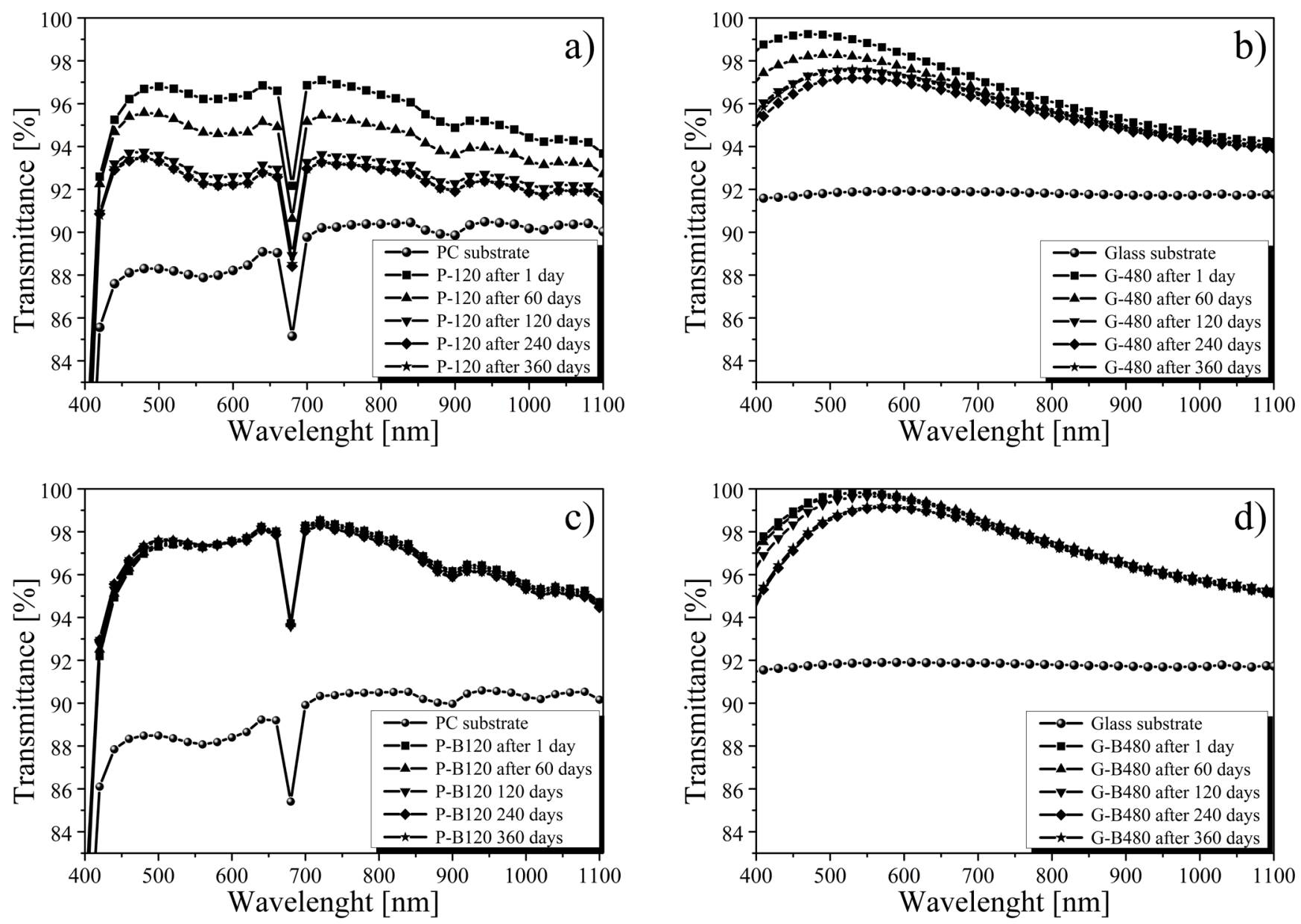

Fig. 6 Transmittance spectra of the bare $\mathrm{PC}$ and glass substrate and the untreated $\mathrm{SiO}_{2}$ coatings $(\mathrm{P}-120)$ on $\mathrm{PC}(\mathbf{a})$, untreated $\mathrm{SiO}{ }_{2}$ coatings $(\mathrm{G}-480)$ on glass $(\mathbf{b})$, ammonia-treated $\mathrm{SiO}_{2}$ coating (P-B120) on PC (c), and ammonia-treated $\mathrm{SiO}_{2}$ coating (G-B480) on glass (d) after 1, 60, 120, 240, and 360 days of storage. The absorption peak appearing at $682 \mathrm{~nm}$ in (a) and (c) is attributed to the absorption of a UV protection additive found in the PC substrate.

storage) regardless of the network strengthening. The decrease of transmittance values is significant and gradual when no specific treatment was applied (Fig. 7). P-120 and G-480 samples show decreases in the maximum light transmittance of values $3.6 \%$ and $1.6 \%$, respectively (Fig. $7 \mathrm{c}$ and $7 \mathrm{~d}$ ). This decrease was calculated as the difference between the first and the latest measured values of the same samples. The decrease is more significant in the case of P-120 compared to G-480, presumably due to the lower heat treatment temperature $\left(120^{\circ} \mathrm{C}\right)$ used for samples on PC. P-B120 and G-B480 samples, however, kept their high transmittance values $(>98 \%$, and $>99 \%)$ even after one year of storage.

Analysis of transmittance spectra: layer thickness (d), effective refractive index (n), and porosity of silica coatings

The silica coatings were characterized via optical fitting model. Layer thickness and effective refractive index were calculated for the untreated (P-120 and G-480) and ammonia treated (P-B120 and G-B480) silica coatings prepared on PC and glass substrates.

The thickness of untreated samples (P-120, and G-480, Fig. $8 \mathrm{a}$ and $8 \mathrm{~b}$ ) begun to decrease after 30 days. In comparison, the base vapour treatment prevented the shrinkage of the
P-B120 and G-B480 systems. Thus, it appears that the aging effect observed on the untreated samples can be slowed down by network strengthening treatment of the lyogels in ammonia vapour atmosphere.

The effective refractive index values of the samples are presented in Fig. 8c and 8d. Pluronic PE1500 is a water soluble and thermally decomposable surfactant [24], therefore it was removed from the silica coating during water extraction or calcination. The differences in the measured refractive index values can be connected to the effectiveness of the template removal methods. In the case of coatings on PC when moderate temperature heat treatment and extraction was used, the refractive index values measured on the as-prepared samples are high $(1.368 \pm 0.002$ for $\mathrm{P}-120$, and $1.323 \pm 0.002$ for P-B120). In contrast, the higher temperature heat treatment used for coatings on glass resulted in lower refractive index values $(1.300 \pm 0.003$ for $\mathrm{G}-480$, and $1.267 \pm 0.004$ for G-B480). Out of all measured samples, P-B120 samples are unique in that they show a constant refractive index over the entire measured interval (Fig. 8). However, the refractive index values of the untreated P-120 samples reached the refractive index of the bulk silica (1.45) after only 90 days. This rapid 

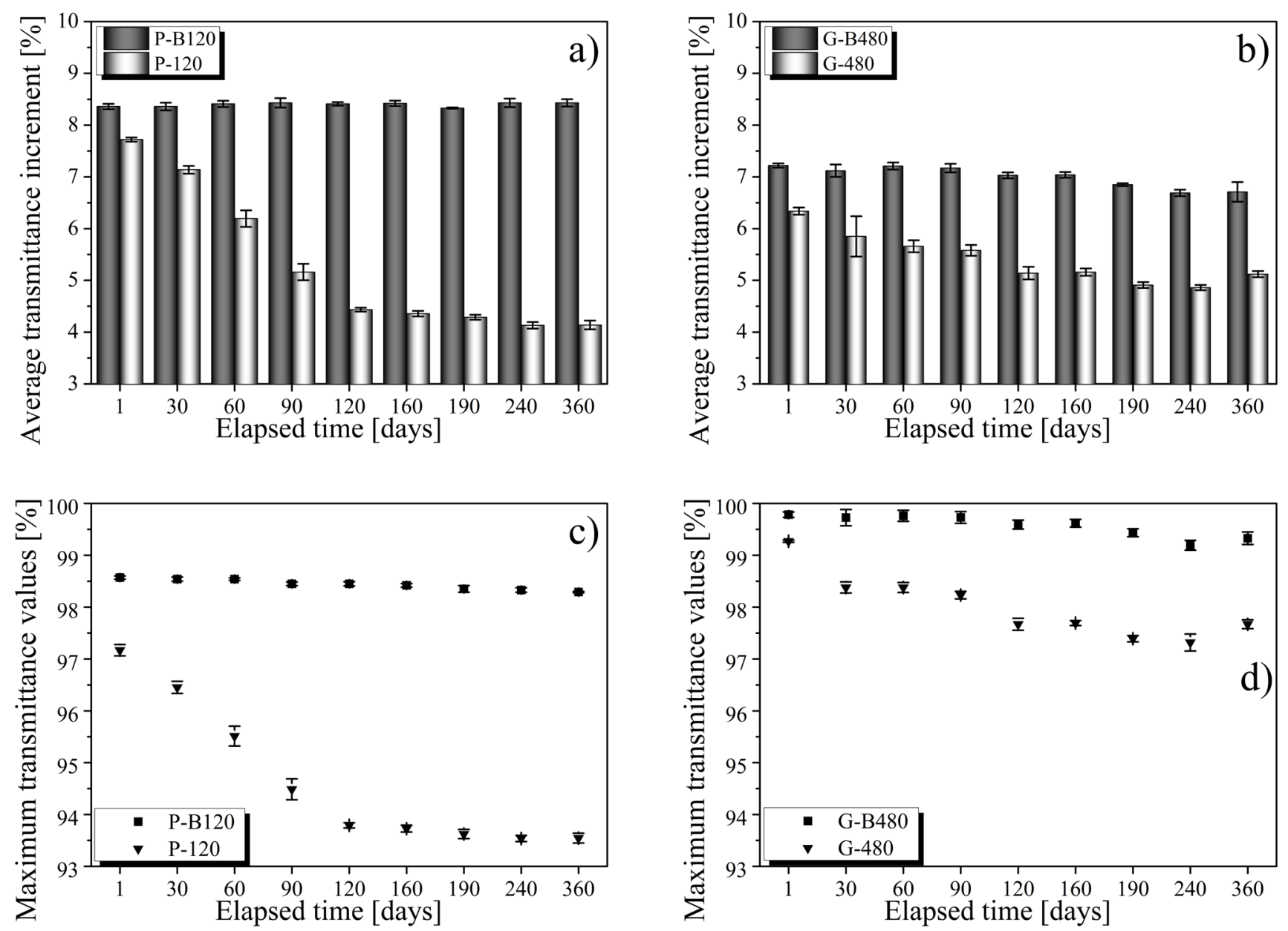

Fig. 7 The average transmittance increment (ATI, a, b) and maximum transmittance $\left(\mathrm{T}_{\max }, \mathbf{c}, \mathbf{d}\right)$ as a function of the elapsed time. Data are shown for untreated (P-120) and ammonia-treated (P-B120) $\mathrm{SiO}_{2}$ coatings on PC substrate (a, c), and untreated (G-480) and ammonia-treated (G-B480) SiO 2 coating on glass substrate (b, d) obtained by UV-Vis spectrophotometry during 360 days.

Table 4 The porosity values of untreated (P-120, G-480) and ammonia-treated (P-B120, G-B480) coatings on PC and glass substrate obtained by UV-Vis spectrophotometry during 360 days. 120 and 480 refers to the temperature of heat treatment.

\begin{tabular}{lccccccccc}
\hline Porosity [\%] & $\mathbf{1}$ day & $\mathbf{3 0}$ days & $\mathbf{6 0}$ days & $\mathbf{9 0}$ days & $\mathbf{1 2 0}$ days & $\mathbf{1 6 0}$ days & $\mathbf{1 9 0}$ days & $\mathbf{2 4 0}$ days & $\mathbf{3 6 0}$ days \\
\hline P-120 & 18 & 13 & 7 & 0 & 0 & 0 & 0 & 0 \\
P-B120 & 27 & 27 & 27 & 28 & 27 & 27 & 26 & 27 \\
G-480 & 32 & 28 & 29 & 28 & 28 & 24 & 23 & 22 & 25 \\
G-B480 & 39 & 37 & 39 & 38 & 36 & 36 & 35 & 34 & 34 \\
\hline
\end{tabular}

increase in the refractive index is attributed to the collapsing of the pore system (ca. 20\% decrease in coating thickness can also be observed). In case of the untreated samples on glass substrates, although the refractive index values increased in time, this increase was not significant (Fig. 8d) presumably due to the high temperature $\left(480^{\circ} \mathrm{C}\right)$ heat treatment. Helsch et al. have described the spontaneous migration of ions in glass toward the coating, due to the varied composition of glass $\left(\mathrm{Na}_{2} \mathrm{O}, \mathrm{K}_{2} \mathrm{O}\right.$, $\mathrm{CaO}, \mathrm{MgO})$ [25]. The high temperature during heat treatment helps the migration, these ions fill the porous system, which results in the increase of the refractive index values.

Porosity values calculated with the help of the effective refractive index values of the layers and the refractive index values of the bulk silica $\left(\mathrm{n}_{\text {bulk }}=1.46\right)$ and air $\left(\mathrm{n}_{\text {air }}=1.00\right)$ are listed in Table 4. The as-prepared untreated G-480 samples show higher porosity $(32 \%$, Table 4$)$ than the P-120 samples (18\%, Table 4). G-B480 and G-B120 samples kept their high porosity and only a moderate decrease can be observed in the measured interval. In contrast, P-120 samples no longer appear to be porous after 60 days. In accordance with their refractive index values, the P-B120 samples show constant porosity throughout 60 days, while a decrease in the porosity values was observed for all other samples. From these results, it can be concluded that porous silica coatings network strengthened in lyogel form with ammonia vapours are more stable on PC substrate (P-B120) than on glass substrate (G-B120). 

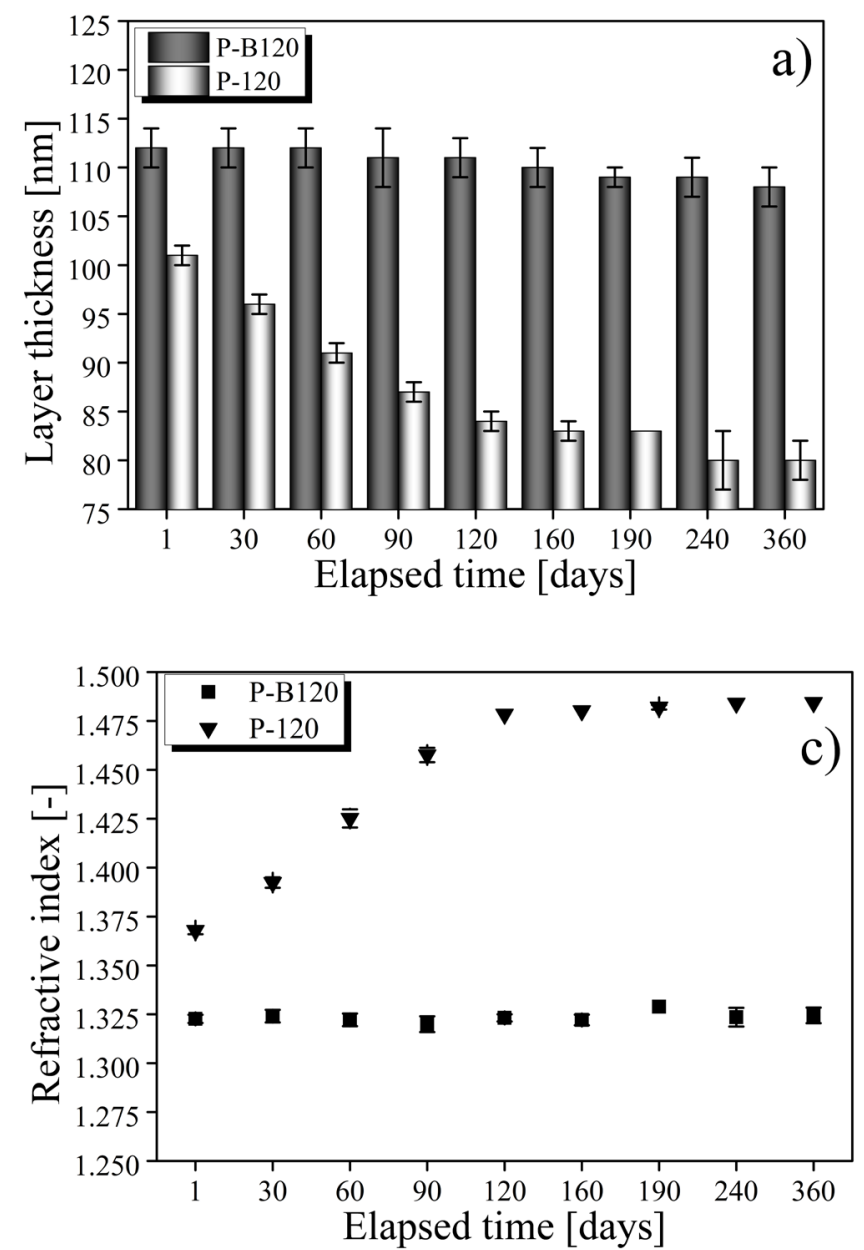
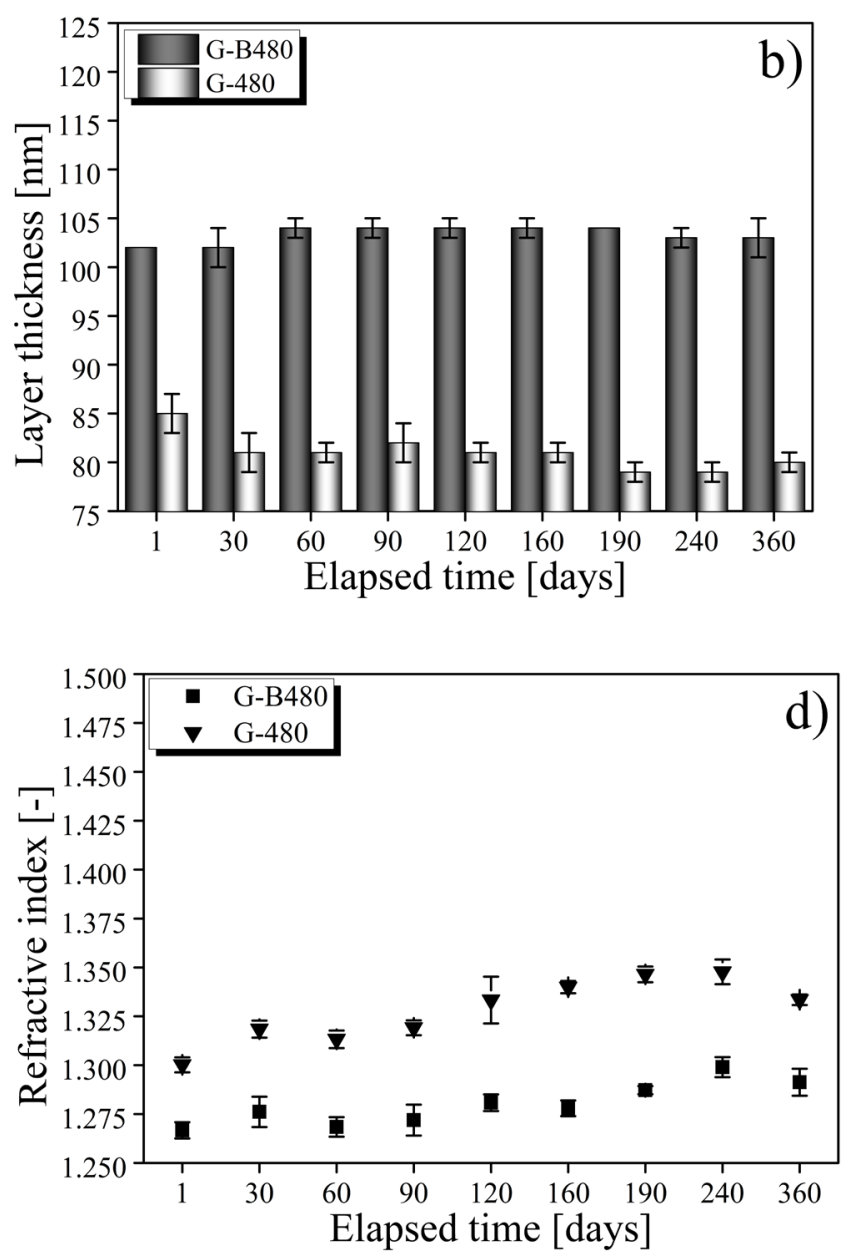

Fig. 8 The silica layer thickness $(\mathbf{a}, \mathbf{b})$ and effective refractive index (c, d) values of untreated (P-120) and ammonia-treated (P-B120) SiO ${ }_{2}$ coatings on $\mathrm{PC}$ substrate (a, c), and untreated (G-480) and ammonia-treated (G-B480) $\mathrm{SiO}_{2}$ coating on glass substrate (b, d) obtained by the analysis of UV-Vis transmittance spectra during 360 days.

\section{Conclusion}

The results reported show that without network strengthening the shrinkage of the layers is responsible for the temporal decrease of light transmittance. The sorption of water vapour and possible pollutants present in air only causes a small decrease in the light transmittance, in spite of the completely open pore structure of the coatings.

The network strengthening treatment carried out in ammonia vapour proved the most efficient at inhibiting the shrinkage. The hydrochloric acid vapour treatment showed only moderate results. A possible explanation for this is that during acid catalysis there is no considerable increase in the reaction rate of polycondensation, which is responsible for the network strengthening. The ammonia treatment has proven most advantageous to use on the coatings still containing pore structuring templates. According to our results, the ammonia treatment administered after the removal of the templates causes considerable shrinkage in the coatings, which decreases the light transmittance. According to our hypothesis, the pores still containing templates are not able to shrink in ammonia vapour atmosphere because the surfactant micelles act as an opposing force against the compressing pore walls.
It was shown that in case of optimal ammonia vapour treatment ( 1 and 3 hours in the vapours of $2 \mathrm{M}$ ammonia solution) in the investigated wavelength range $(410-780 \mathrm{~nm})$ the average transmittance increment only changes within the margin of error for PC substrates during one year (value of $8.4 \%$ ), while in the case of glass surfaces there is significant decrease after 160 days (from 7.7\% to 4.1\%). According to our hypothesis, this effect is related to the temporal instability (due to the migration of components) of the substrate (glass). The decrease of maximum transmittance is also lower in case of PC substrates: $0.28 \%$ for PC and $0.45 \%$ for glass.

\section{Acknowledgement}

The authors thank Olga Krafcsik, Erzsébet Hild, Judit Tormási, Martin Schneider, Dániel Szilárd Varga for their help in this work.

This project has received funding from the European Union's Horizon 2020 research and innovation program under grant agreement No 683541. The research work has been accomplished in the framework of the „BME R+D+I project”, supported by the grant TÁMOP 4.2.1/B-09/1/KMR-2010-0002.

Emöke Albert's research work was supported by the European Union and the State of Hungary, co-financed by the 
European Social Fund in the framework of TÁMOP-4.2.4.A/ 2-11/1-2012-0001 "National Excellence Program".

\section{References}

[1] Zhu, J., Yu, Z., Fan, S., Cui, Y. "Nanostructured photon management for high performance solar cells." Materials Science \& Engineering R. 70(3-6), pp. 330-340. 2010.

https://doi.org/10.1016/j.mser.2010.06.018

[2] Chen, D. "Anti-refection (AR) coatings made by sol-gel processes: A review." Solar Energy Materials \& Solar Cells. 68(3-4), pp. 313-336. 2001. https://doi.org/10.1016/S0927-0248(00)00365-2

[3] Raut, H. K., Ganesh, V. A., Nairb, A. S., Ramakrishna, S. "Anti-reflective coatings: A critical, in-depth review." Energy \& Environmental Science, 4(10), pp. 3779-3804. 2011.

https://doi.org/10.1039/C1EE01297E

[4] Hedayati, M. K., Elbahri, M. "Antireflective Coatings: Conventional Stacking Layers and Ultrathin Plasmonic Metasurfaces, A Mini-Review." Materials. 9(6), pp. 497-519. 2016.

https://doi.org/10.3390/ma9060497

[5] Thomas, I. M. "Effect of binders on the damage threshold and refractive index of coatings prepared from colloidal suspensions." In: Proceedings of the SPIE. 24th Annual Boulder Damage Symposium Proceedings. Laser-Induced Damage in Optical Materials, Vol. 1848, pp. 281-289. 1993. https://doi.org/10.1117/12.147405

[6] Thomas, I. M., Burnham, A. K., Ertel, J. R., Frieders, S. C. "Method for reducing the effect of environmental contamination of sol-gel optical coatings." In: Proceedings of the SPIE. Third International Conference on Solid State Lasers for Application to Inertial Confinement Fusion, Vol. 3492, pp. 220-229. 1999.

https://doi.org/10.1117/12.354236

[7] Li, X., Shen, J. "The stability of sol-gel silica coatings in vacuum with organic contaminants." Journal of Sol-Gel Science and Technology. 59(3), pp. 539-545. 2011.

https://doi.org/10.1007/s10971-011-2524-6

[8] Hong, T., Lei, Z., Yao, X., Dong, W., Zhong-Hua, W., Hai-Bing, L. U., Xiao-Dong, Y. "Comparision of Silica Anti-Reflective Films Obtained via a Sol-Gel Process in the Presence of PEG or PVP." Acta Physico-Chimica Sinica. 28(5), pp. 1197-1205. 2012. https://doi.org/10.3866/PKU.WHXB201202231

[9] Belleville, P. F., Floch, H. G. "Ammonia-hardening of porous silica antireflective coatings." In: Proceedigns of the SPIE. Sol-Gel Optics III. Vol. 2288, pp. 25-32. 1994. https://doi.org/10.1117/12.188957

[10] Li, X., Gross, M., Oreb, B., Shen, J. "Increased Laser-Damage Resistance of Sol - Gel Silica Coating by Structure Modification." The Journal of Physical Chemistry C. 116(34), pp. 18367-18371. 2012. https://doi.org/10.1021/jp307390u

[11] Boudot, M., Gaud, V., Louarn, M., Selmane, M., Grosso, D. "Sol-Gel Based Hydrophobic Antireflective Coatings on Organic Substrates: A Detailed Investigation of Ammonia Vapour Treatment (AVT)." Chemistry of Materials. 26(5), pp. 1822-1833. 2014. https://doi.org/10.1021/cm403787v

[12] Li, C., Wilkes, G. L. "The Mechanism for 3Aminopropyltriethoxysilane to Strengthen the Interface of Polycarbonate Substrates with Hybrid Organic-Inorganic Sol-Gel Coatings." Journal of Inorganic and Organometallic Polymers. 7(4), pp. 203-216. 1997. https://doi.org/10.1023/A:1021690423544
[13] Hild, E., Deák, A., Naszályi, L., Sepsi, Ö., Ábrahám, N., Hórvölgyi, Z. "Use of the optical admittance function and its WKB approximation to simulate and evaluate transmittance spectra of graded-index colloidal films." Journal of Optics A: Pure and Applied Optics. 9(10), pp. 920-930. 2007. https://doi.org/10.1088/1464-4258/9/10/023

[14] Volentiru, E., Nyári, M., Szabó, G., Hórvölgyi, Z., Mureşan, L. M. "Silica sol - gel protective coatings against corrosion of zinc substrates." Periodica Polytechnica Chemical Engineering. 58(S2014), pp. 61-66. 2013. https://doi.org/ https://doi.org/10.3311/PPch.7302

[15] Albert, E., Cotolan, N., Nagy, N., Sáfrán, G., Szabó, G., Mureşan, L. M., Hórvölgyi, Z. "Mesoporous silica coatings with improved corrosion protection properties." Microporous and Mesoporous Mateials. 206, pp. 102-113. 2015.

https://doi.org/10.1016/j.micromeso.2014.12.021

[16] Press, W. H., Teukolsky, S. A., Vetterling, W. T., Flannery, B. P. "Numerical Recipes in C - The Art of Scientific Computing." Cambridge University Press, 1992.

[17] Lorenz, L. "Ueber die Refractionsconstante." Annalen der Physik. 247(9), pp. 70-103. 1880. (in German) https://doi.org/10.1002/andp.18802470905

[18] Lorentz, H. A. "The theory of electrons and its applications to the phenomena of light and radiant heat." Leipzig: B.G. Teubner; New York: G.E. Stechert, 1916.

[19] Grigoriev, D., Gorin, D., Sukhorukov, G. B., Yashchenok, A., Maltseva, E., Möhwald, H. "Polyelectrolyte/magnetite Nanoparticle Multilayers: Preparation and Structure Characterization." Langmuir. 23(24), pp. 12388-12396. 2007. https://doi.org/10.1021/la700963h

[20] Mogilnikov K. P., Baklanov, M. R. "Determination of Young's Modulus of Porous Low-k Films by Ellipsometric Porosimetry." Electrochemical and Solid-State Letters. 5(12), pp. F29-F31. 2002. https://doi.org/10.1149/1.1517771

[21] Lu, Y., Penzkofer, A. "Absorption behaviour of methanolic rhodamine 6G solutions at high concentration." Chemical Physics. 107(2-3), pp. 175-184. 1986.

https://doi.org/10.1016/0301-0104(86)85002-9

[22] Albert, E., Basa, P., Deák, A., Németh, A., Osváth, Z., Sáfrán, G., Zolnai, Z., Hórvölgyi, Z., Nagy, N. "Introducing nanoscaled surface morphology and percolation barrier network into mesoporous silica coatings." RSC Advances. 5, pp. 60041-60053. 2015. https://doi.org/10.1039/c5ra09357k

[23] Vogel, R., Meredith, P., Harvey, M. D., Rubinsztein-Dunlop, H. "Absorption and fluorescence spectroscopy of rhodamine $6 \mathrm{G}$ in titanium dioxide nanocomposites." Spectrochimica Acta Part A-Molecular and Biomolecular Spectroscopy. 60(1-2), pp. 245-249. 2004. https://doi.org/10.1016/S1386-1425(03)00218-X

[24] Gallet, G., Carroccio, S., Rizzarelli, P., Karlsson, S. "Thermal degradation of poly (ethylene oxide - propylene oxide - ethylene oxide) triblock copolymer : comparative study by SEC / NMR, SEC / MALDI-TOF-MS and SPME /GC-MS." Polymer. 43(4), pp. 1081-1094. 2002. https://doi.org/10.1016/S0032-3861(01)00677-2

[25] Helsch, G., Radlein, E., Frischat, G. H. "On the origin of the aging process of porous $\mathrm{SiO} 2$ antirefection coatings." Journal of Non-Crystalline Solids. 265(1-2), pp. 193-197. 2000. https://doi.org/10.1016/S0022-3093(99)00880-7 